\title{
Research on Fault Self-healing Method of Smart Distribution Network Based on Binary Hybrid Algorithm
}

\author{
Wang Baoyi ${ }^{\text {a }}{ }^{*}$, Qian Yalun ${ }^{b}$, Zhang Shaomin ${ }^{c}$ \\ School of Control and Computer Engineering, North China Electric Power University, \\ Baoding,Hebei Province,China \\ a.email:wangbaoyi@126.com, b.email:,qianyalun@qq.com,c.email:zhangshaomin@126.com
}

Keywords: Fault Self-healing, Smart Distribution Network, Binary Quantum Particle Swarm Algorithm, Binary Fruit Fly Optimization Algorithm, Binary Hybrid Algorithm

Abstract: In this paper, the mathematical model of fault self-healing with the minimum net loss and node voltage deviation is proposed. Then, against the deficiencies of the fruit fly optimization algorithm, combine binary quantum particle swarm algorithm and binary fruit fly optimization algorithm to produce a binary hybrid algorithm for fault self-healing process. Finally, the experiment proved that the algorithm has faster convergence rate and stronger global optimization ability.

\section{Introduction}

With the speedy development of the economy, people's demand on the electricity is rapidly increased, while requirements on the quality and reliability of power supply are significantly improved. Distribution network is directly connecting with its user, so the occurrence of its failure will inevitably have a huge impact on people's life ${ }^{[1]}$. The core function of smart distribution network called self-healing can effectively reduce the occurrence of large-scale blackout and provide an important guarantee for the efficient, safe and stable operation of distribution network.

At home and abroad, scholars have made a lot of research on the fault self-healing method of distribution network, which can be divided into three types: mathematical optimization method ${ }^{[2]}$, heuristic search method ${ }^{[3-5]}$ and artificial intelligence method ${ }^{[6-9]}$. In [5], a self-healing method based on heuristic rules and artificial immune is proposed for distribution network. The algorithm's computation is fast and it can effectively solve multi-objective problem. In [6], considering the influence of distributed generation on power grid, a kind of simulated annealing algorithm is used to recover a fault. In [7-9], particle swarm algorithm and improved ant colony algorithm are used to recover the fault. Although the global search ability has been improved to a certain extent, the convergence rate is still limited. 
This paper proposed a smart distribution network fault self-healing mathematical model and a binary hybridization method to get the best fault self-healing scheme. Finally, an example is given to show that the algorithm has better convergence and global optimization ability.

\section{Relevant Algorithm}

\subsection{Binary Quantum Particle Swarm Algorithm}

Quantum particle swarm optimization algorithm (QPSO) is proposed by Sun Jun, Xu Wenbo et al. combining with quantum evolutionary algorithm ${ }^{[10]}$. In view of switch status values in fault self-healing of smart distribution network are a set of discrete variables, so it is necessary to adopt a binary coding method to QPSO (BQPSO).

In the BQPSO algorithm, binary codes 0 and 1 are used to represent position information of the particles, and the Hamming distance is used to describe the distance between the particles ${ }^{[8][11]}$. Then, the population average optimal position mbest $(t)$ is obtained from all the individuals optimal position strings of in binary coded. If the number of 1 in these strings is more than 0 , then the value is 1 , otherwise is $0^{[8][12]}$.

The algorithm steps are described as following:

(1)Initialization: use a string of binary number to represent initial position of each particle.

(2)Calculate current fitness function values for each particle and compare with the previous.

(3)Calculate the value of the population average optimal position.

(4)Calculate the current global optimal position and compare it with the previous.

(5)Calculate the local attraction factor to generate a new population;

(6)Determine whether reach the maximum number of iteration or termination conditions, if not, then go to step (2), otherwise the algorithm is over.

\subsection{Binary Fruit Fly Optimization Algorithm}

Fruit Fly Optimization Algorithm (FOA) is a new group intelligent method for seeking global optimization based on the evolution of fruit fly foraging behavior proposed by Professor Pan Wenchao of Taiwanese scholar in June 2011 ${ }^{[13]}$.

To get close to the food source, set the random direction and distance of each fruit fly near to the food source as (1).And location update equation is (2).

$$
\begin{array}{r}
X_{i}=X+\operatorname{random}() \\
X_{i j}(t+1)=X_{i j}(t)+\alpha \cdot L(\lambda)
\end{array}
$$

The expression of the taste concentration determination value of each fruit fly can be described as (3) and (4). Then calculate the taste concentration of each fruit fly as (5). While it is necessary to record the position of the fruit fly with the highest taste concentration value, and fly to it by visual.

$$
\text { Dist }_{i}=\sqrt{X_{i}^{2}+Y_{i}^{2}}
$$




$$
\begin{array}{r}
S_{i}=\frac{1}{\text { Dist }_{i}} \\
\text { smell }_{i}=\operatorname{SmellFunction}_{\left(S_{i}\right)}
\end{array}
$$

At last, The following method is used to achieve the binary coding of the algorithm as (6), where $\delta$ is called control factor, and its range is $[0,1]$

$$
\begin{aligned}
& \text { IF } \operatorname{rand}() \leq \delta \\
& \operatorname{Sigmoid}(\text { step })=\frac{1}{1+e^{- \text {step }}} \\
& \left\{\begin{array}{l}
x_{i j}(t)=1, \text { rand }() \leq \text { Sigmoid }(\text { step }) \\
x_{i j}(t)=0, \text { else }
\end{array}\right.
\end{aligned}
$$

ELSE

$$
\begin{aligned}
& \text { IF } \quad \text { step } \leq 0 \text { : } \\
& \text { Sigmoid(step })=1-\frac{2}{1+e^{- \text {step }}} \\
& \left\{\begin{array}{l}
x_{i j}(t+1)=0, \text { rand }() \leq \text { Sigmoid }(\text { step }) \\
x_{i j}(t+1)=x_{i j}(t), \text { else }
\end{array}\right. \\
& \text { IF step }>0 \text { : } \\
& \operatorname{Sigmoid}(\text { step })=\frac{2}{1+e^{-s t e p}}-1 \\
& \left\{\begin{array}{l}
x_{i j}(t+1)=1, \text { rand }() \leq \operatorname{Sigmoid}(\text { step }) \\
x_{i j}(t+1)=x_{i j}(t), \text { else }
\end{array}\right.
\end{aligned}
$$

\section{Fault Self-healing Mathematical Model of Smart Distribution Network}

\subsection{Objective Function}

In this paper, the net loss and the node voltage bias are used as the two sub-functions of the objective function, and the two functions' weights are given by the linear weighting method, as equations (7). Finally, the final total objective function is obtained by linearly weighted summing. And then, we can obtain fault self - healing mathematical Model of smart Distribution Network with DG.

$$
\min F(x)=\min \lambda_{1} f_{1}+\lambda_{2} f_{2}=\min \sum x_{i} R_{i}\left|I_{i}\right|^{2}+\left|V_{i}-V_{r i}\right|
$$

Where $\lambda_{1}$ and $\lambda_{2}$ are the weighting factors, $x_{i}$ represents the state of the switch node $i$, $x_{i}=1$ means it is closed, $x_{i}=0$ indicates $\mathrm{I}$ is disconnection, $R_{i}, I_{i}, V_{i}$ and $V_{r i}$ respectively denote the resistance, current, actual voltage and Rated voltage of branch I.

\subsection{Restrictions}

(1) Equality constraint: the power demand of node $t$ is equal to the difference between inflow 
power and outflow power.

$$
\sum_{i \in I E_{t}} S_{i t}-\sum_{j \in O E_{t}} S_{j t}=P_{t}, t=1,2, \ldots, m
$$

(2) Network topology always maintains radial, and has no ring network.

(3) Inequality constraints:

(1)branch current constraint:

$$
I_{i} \leq I_{i \max }
$$

(2)Node voltage constraint:

$$
U_{i \min } \leq U_{i} \leq U_{i \max }
$$

(3)Feeder capacity constraint:

$$
S_{i} \leq S_{i \max }
$$

(4) Distributed generation capacity constraint:

$$
P_{i_{-} D G}^{2}+Q_{i_{-} D G}^{2} \leq S_{i_{-} D G_{-} \max }^{2}
$$

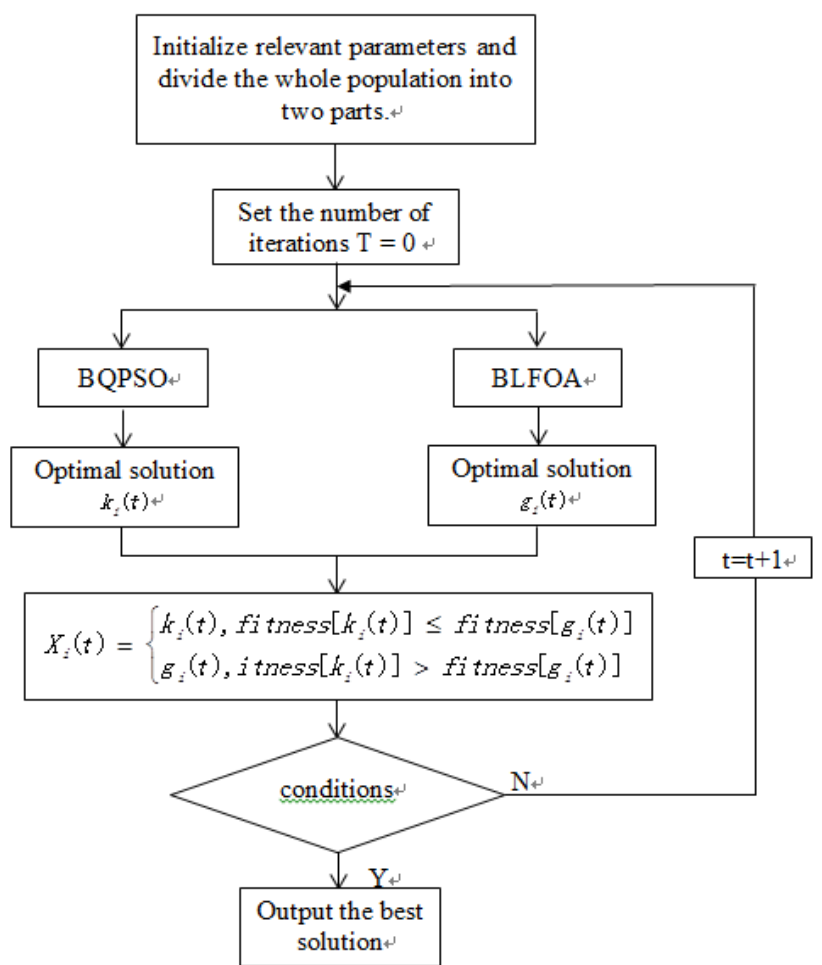

Figure 1 The basic procedure of binary hybrid algorithm (BQPLF)

\section{Fault Self-healing Method Based on a Binary Hybrid Algorithm}

LBFOA algorithm is easy to get up early and fall into the local optimal solution, but its global optimization ability is strong. BQPSO algorithm can cover all of feasible solutions, so as to avoid the occurrence of precocious phenomena and make it difficult to fall into the local optimal solution. 
In the light of it, we mix them by using two-group evolution strategy and information interaction mechanism to get a binary hybrid algorithm (BQPLF) as figure 1. Then use it to optimize the fault self-healing of DG-containing smart distribution network, and the optimal recovery strategy is found with the minimum net loss and node voltage deviation.

\section{Case Study}

Take IEEE33 node distribution network system as an example in Figure 2. Only the capacity of the DG installed on the 18-th node is 250kVA and others are 500kVA. Each DG's power factor is set to 0.8 . Assuming a permanent fault occurs between the nodes 1 and 2 , and choose $\lambda_{1}=0.8, \lambda_{2}=0.2$.

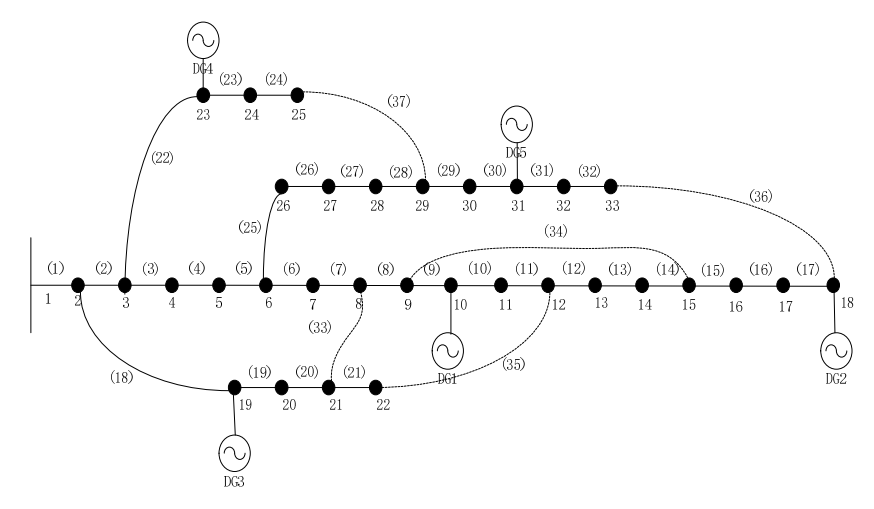

Figure 2 IEEE 33 node distribution network system

Table 1 The failure heal solution in IEEE 33 node distribution network system

\begin{tabular}{|c|c|c|}
\hline switch operation sequence & switch operation number & $\begin{array}{c}\text { isolated island } \\
\text { number }\end{array}$ \\
\hline $\begin{array}{c}\text { open (5) (7) (15) (17) (23) (29), close } \\
(35)(36)\end{array}$ & 8 & 2 \\
\hline
\end{tabular}

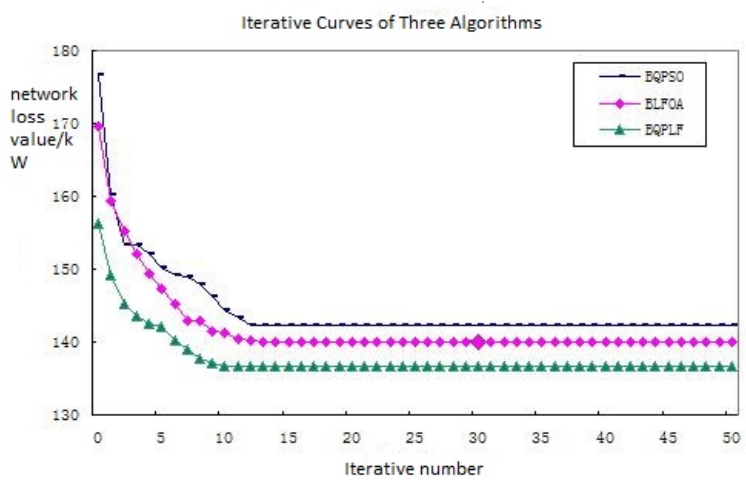

Figure 3 The iterative curves of three algorithms

Using the binary hybrid algorithm proposed in this paper to recover the fault, and the scheme 
obtained is shown in Table1. In the end, the iterative curve of these algorithms is shown in Figure 3. Obviously, BQPLF's convergence speed is faster than others, and the global search ability is stronger.

\section{Conclusions}

This paper first proposed the mathematical model of fault self-healing. Then, the binary hybrid algorithm (BQPLF) is proposed by mixing binary quantum particle swarm algorithm and binary fruit fly optimization algorithm. Then use it to recover the smart distribution network with distributed generation to obtain the optimal fault recovery scheme. Finally, take the IEEE33 node distribution system as an example, the validity of the BQPLF algorithm is proved, and it also has fast convergence speed and strong global optimization ability.

\section{References}

[1] Li Dapeng. Study of Self-healing Techniques for Smart Distribution Networks with DGs[D]. Tianjin University, 2012.

[2] Zhenzhi Lin, Fushuan Wen, Yusheng Xue. A Restorative Self-Healing Algorithm for Transmission Systems Based on Complex Network Theory [J].IEEE Transactions on Smart Grid, 2016, 7: 2154-2162.

[3] Pang Qingle, Gao Houlei, Li Tianyou. Load Balancing Based Fault Service Restoration for Smart Distribution Grid[J]. Power System Technology, 2013, 02: 342-348.

[4] Fazel Abbasi and Seyed Mehdi Hosseini. Optimal DG allocation and sizing in presence of storage systems considering network configuration effects in distribution systems [J].IET Generation, Transmission \& Distribution, 2016, 10: 617-624.

[5] Liu Kai, Li Wenpei, Cheng Qianshan et al. Self-healing of smart distribution network based on heuristic rules and artificial immune [J].Journal of Wuhan University (Engineering Science), 2016,02: 218-222.

[6] XIANG Yinxing, WU Wenxuan, LIN Han, and et al. Self-healing control and reconstruction optimization of smart grid with distributed generation[J]. Electrical Applications, 2015, 18: 68-71.

[7] Yao Yuhai, Wang Zengping, Guo Kunya et al. Multi-objective binary particle swarm optimization algorithm based on E-dominated solution for fault recovery of distribution network [J]. Power System Protection and Control, 2014, 23: 76-81.

[8] Zhang Tao, Shi Suyi, Xu Xueqin. Reconstruction of distribution network with distribution generation based on binary quantum particle swarm optimization [J].Power System Protection and Control, 2016, 04: 22-28.

[9] Xu Qiang,Guo Haibin,Feng Xugang et al.Research on self-healing strategy of smart distribution grid based on improved ant colony algorithm[C].2016 Chinese Control and Decision Conference (CCDC),2016:390-395.

[10] Li Yang. Research on Reactive Power Optimization Based on Multi - Agent Quantum Particle Swarm Optimization [D].GuangDong University of Technology, 2014.

[11] Zhang Mingguang, Wang Shiliang. Multi-objective Optimization Planning of Power Grid Based on BQPSO [J]. Computer Engineering and Applications, 2015,18: 266-270.

[12] Wang Shiliang. Multi-objective optimization planning of smart grid based on improved quantum particle swarm optimization [D]. Lanzhou University of Technology, 2014.

[13] Ling Feihong, Xue Yuqiang, Lin Minghan. Application of FOA algorithm in distribution network reconfiguration [J]. Electrical switch, 2016, 54 (02): 87-90 + 96 [2017-09-27]. 